\title{
Optimal operation of electric vehicle charging station in an open electricity market
}

\author{
Ahmad Abuelrub $^{\mathrm{a}^{*}}$, Abdel Rahman Al khalayleh $^{\mathrm{a}}$, Ahmad Allabadi $^{\mathrm{a}}$ \\ ${ }^{a}$ Jordan University of Science and Technology, Irbid 22110, Jordan
}

\begin{abstract}
In this paper, a multi-mode optimization technique for Electric Vehicle (EV) unidirectional charging station is presented. The objective of the proposed system is to reduce the cost of EV charging in an open electricity market. Moreover, the proposed algorithm can be integrated with renewable resources such as photovoltaics (PV), and wind turbines among others. The optimization procedure has three modes of operation to suit customer needs under different circumstances. The three suggested modes of operation are normal mode, eco mode, and boost mode. The aforementioned technique aims to reduce the cost of EV charging by finding the optimal schedule for charging in the available time horizon in each mode. In addition, the optimization algorithm varies the charging rate in order to meet the desired target for some special cases. The optimization technique is based on the Priority List which makes it simple to be implemented online. A case study is presented to illustrate the benefits of using the suggested optimization procedure. The results show the financial feasibility of the proposed procedure.
\end{abstract}

Keywords: Electric vehicle, charging station, optimization, photovoltaic

\section{Introduction}

Transportation electrification is expected to proliferate in the foreseeable future, due to the fact that EVs have zero greenhouse gas emissions [1]. In addition, the employment of renewable sources can be used to handle energy scarcity [2]. Therefore, charging stations for EVs which use hybrid energy systems (i.e. systems consist of renewable resources such as PV and conventional sources such as energy market) are needed. The use of renewable energy sources is important to reduce environmental pollution, but these sources are intermittent by nature. Therefore, they are usually combined with a conventional one in order to make overall system operation uninterruptable. However, the operation of such a system is challenging and needs careful study to make its operation optimal, thus reducing the EVs charging cost.

Charging station operation is heavily discussed in published literature. In [3], a four-stage optimization algorithm was proposed for the purpose of reducing charging cost while integrating a PV system within the charging station. A charging method was proposed for EV charging facilities in response to Time-OfUse (TOU) price in [4]. In [5], the authors investigated the effect of fast-charging EV on an existing utility company distribution system. Power-flow, short-circuit, and protection analysis were performed using utility-grade software packages. An advanced system design for a fuel-cell-powered batterycharging station with three control strategies for active power sharing among batteries was presented in [6]. The authors of [7] designed and exploited a supervisory control system for commercial electric vehicle charging station based on virtual distributed processing unit technology of distributed control system. However, all the aforementioned references only consider charging stations. Therefore, the contribution of this paper is to design a simple optimization algorithm for residential charging points to reduce charging costs.

Increased usage of EVs raises the demand for energy while unorganized EV charging increases the stress on the power system and elevates the cost of energy in open electricity markets since open

\footnotetext{
* Manuscript received May 26, 2018; revised May 24, 2019.

Corresponding author. Tel.: +962792907358; E-mail address: amabuelrub@just.edu.jo.

doi: 10.12720/sgce.8.4.495-499
} 
electricity markets are characterized by the hourly update of energy prices depending on the supply and demand interactions. This paper solves these concerns taking into account that around $90 \%$ of the time, on average, passenger vehicles are parked and this time period of inactivity is longer than the time needed for fully charging the battery of the electric vehicle [8]. The suggested charging station system is shown below in Fig. 1. We utilize PVs here since PVs are the dominant method of alleviating the drawbacks of fuel-based greenhouse gas emissions. This will help the environment recover from the tremendous amount of greenhouse gas emissions current present. Energy storage is used to store the electrical energy generated from the PV plant in case of applying the off-grid charging mode. The grid will be utilized through the mains to absorb the power as well as to export it, and therefore, overall system operation will be continuous. DC-DC power converters have recently developed to a level that will enable using a multiinput power converter to help implement the suggested system.

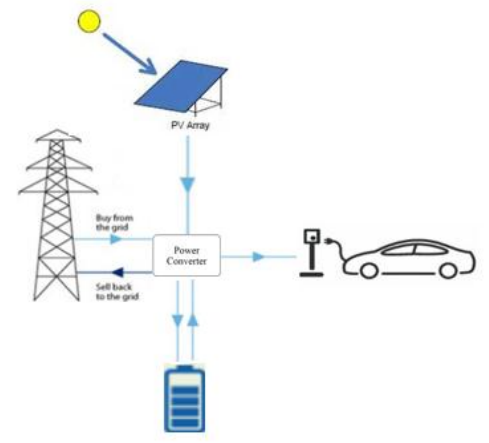

Fig. 1. Proposed charging station system.

In the following discussion, the proposed method is employed for on-grid system considering existence or absence of the PV system. Moreover, this work is extended to be applied for an off-grid PV system integrated with the smart charging technique since there is a maximum limit for the gridconnected PV systems capacity attached to distribution transformer [9]. Hence, a considerable number of residents will not be able to utilize the renewables.

\section{Problem Formulation}

In this section, the problem is presented as an optimization problem that minimizes the EV charging cost.

\subsection{Problem description}

In open electricity markets, prices severely fluctuate even within the same day. The price of electrical energy within an arbitrary day at peak time is around three times the price of the off-peak time [10]. Even with the integration of PV systems, unorganized charging will limit its benefits. Therefore, this paper suggests an operation algorithm to charge EV with the lowest possible cost using priority list optimization algorithm.

\subsection{Optimization algorithm}

With the help of the magnitude of information available online, it is possible to obtain input data for the algorithm such as day-ahead market (DAM) price data and PV-generation data. The optimization technique is illustrated in the following steps:

1) Record the plug-in time $\left(\mathrm{T}_{0}\right)$ and the initial state of charge of the EV battery $\left(\mathrm{SOC}_{\mathrm{in}}\right)$.

2) Specify the final state of charge $\left(\mathrm{SOC}_{\mathrm{fn}}\right)$ and the time of the EV delivery $\left(\mathrm{T}_{\mathrm{f}}\right)$.

3) Use the priority list optimization technique, shown below, to build the optimal schedule and rate of charging of the EV within the available time frame. 
The optimization procedure is illustrated in the flowchart shown in Fig. 2. The flowchart explains the general steps needed to execute the charging operation.

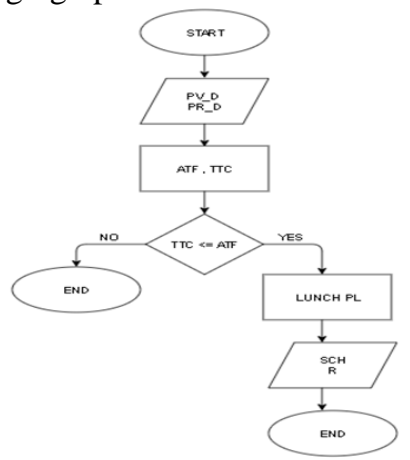

Fig. 2. Flowchart of the proposed operation algorithm.

In Fig. 2, PV_D is the PV data, PR_D is the grid price data, ATF is the available time frame, TTC is the time required to charge EV battery, PL is the priority list, $\mathrm{SCH}$ is the optimal schedule to charge the $\mathrm{EV}$, and $\mathrm{R}$ is the optimal charging rate.

Now, to meet the vast majority of the customer needs, three modes are adapted as follows:

\subsubsection{Normal mode}

The philosophy of operation in this mode is to utilize available timeslots to charge the EV as much as possible. As a user, you are only required to enter the $\mathrm{SOC}_{\mathrm{fn}}$ and the $\mathrm{T}_{\mathrm{f}}$. Then, the optimizer will use this data to obtain the time span of charging and the needed time slots for optimal schedule of charging.

\subsubsection{Eco mode}

In this mode, the problem is relaxed from the $\mathrm{SOC}_{\mathrm{fn}}$ and time of delivery constraints and the energy charged to the EV battery is equal to energy generated from the PV system only. Implementing the Eco mode, with PV in both on and off grid cases, will result in free charging as follows:

1) With the on-grid PV system, the amount of energy charged to the vehicle will be limited by the amount of energy generated by the PV system. However, this amount of energy will be purchased at the lowest possible price.

2) With the off-grid PV system, the same philosophy as for the on-grid PV system is applied with the exception that there is no power exchange between the PV system and the grid.

\subsubsection{Boost mode}

In this mode, the goal is to reach the final state of charge with a minimum time. Implementing this mode, with PV in both on and off grid cases, will ensure the minimum time of charging.

1) With the on-grid PV system, priority is given to meeting the desired final state of charge as fast as possible by charging the vehicle at the maximum charge rate.

2) With the off-grid PV system, the same philosophy applies as for on-grid PV system with the priority of maximizing the contribution of the renewable system in charging the EV.

The suggested modes of operation are summarized in Table 1.

Table 1. Summary of the proposed modes of operation.

\begin{tabular}{c|l|l}
\hline \multirow{2}{*}{ Mode } & \multicolumn{1}{|c}{ PV connection to the grid } \\
\cline { 2 - 3 } Normal & $\begin{array}{l}\mid c \\
\text { Purchase the needed energy at the lowest price } \\
\text { available to meet the desired state. }\end{array}$ & $\begin{array}{l}\text { Absorb available energy from PV panels and storage } \\
\text { compensates the shortage from the grid. }\end{array}$ \\
\hline Eco & $\begin{array}{l}\text { Sell the PV output to the grid and purchase the same } \\
\text { amount at the lowest energy price. }\end{array}$ & Charge the EV by the PV output and the storage. \\
\hline Boost & Charge from the grid at maximum rate of charging. & Maximize the contribution of the renewables. \\
\hline
\end{tabular}




\section{Case Study}

To demonstrate the effectiveness of the proposed algorithm, a comparison is made between the (unorganized) typical charging method called "plug and charge" and the proposed modes of operation. The comparison will show the amount of savings or profits obtained by applying these modes.

\subsection{Initializing the simulation}

Price data [11] and PV output power data [12] are collected for the state of California at San Francisco area on April 26, 2018. This data is shown in Fig. 3a and Fig. 3b respectively.

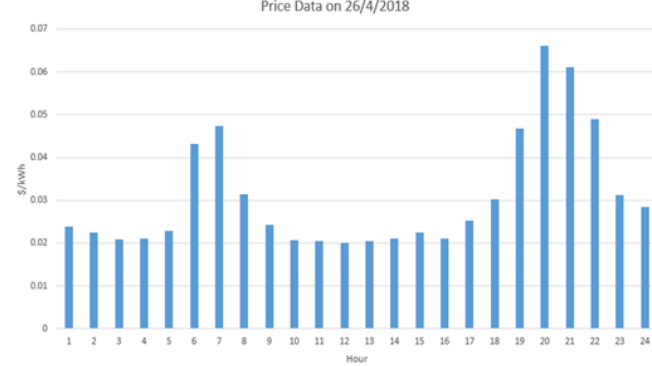

Fig. 3a. Electrical energy price for 24 hours.

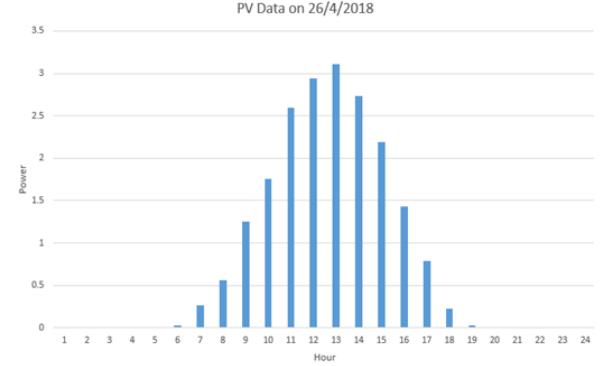

3b. Residential PV system output power for 24 hours

The energy shortage of the EV battery is calculated using (1),

$E_{s}=E_{e v} \times\left(S O C_{f n}-S O C_{i n}\right)$

Where $E_{s}$ is the energy shortage of the EV battery in $\mathrm{kWh}$ and $E_{e v}$ its energy capacity in $\mathrm{kWh}$.

Then, time to charge the battery can be calculated as in (2),

$T T C=\frac{E_{s}}{P_{r}}$

Where $T T C$ is the time to charge the EV battery in $\mathrm{h}$, and $P_{r}$ is the rated power in $\mathrm{kW}$. The usage of constant charging rate at rated power $P_{r}$ from the starting time up to the $S O C_{f n}$ [4] as illustrated in Fig. 5.

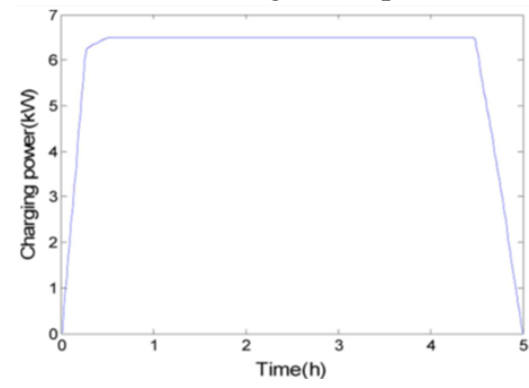

Fig. 5. Charging curve of the lithium-ion battery [4].

\subsection{Simulation results}

The adopted car type here is the Nissan Leaf 2018 [13] with battery energy capacity of $40 \mathrm{kWh}$ and battery rated power of $6.6 \mathrm{~kW}$. The following parameters are chosen for the simulation, $S O C_{i n}=0.2$, $S O C_{f n}=0.8$ (if needed depending on the selected mode), $T_{0}=14: 00$, and $T_{f}=09: 00$ (if needed depending on the selected mode).

The results show the ability to save a considerable amount of money depending on the operating mode. Beginning with normal mode (on-grid), the achieved reduction in cost is $30 \%$ of the cost of typical charging. Moreover, the Eco (on-grid) mode results in 58.4\% of charging the same amount as the Plug \& 
Charge platform. The profits obtained by using the Eco mode here are $0.0366 \$ / \mathrm{h}$. The boost (on-grid) is exactly the same as the typical charger.

For the off-grid system, normal mode results in $79.8 \%$ reduction in cost as compared to the typical charger. Furthermore, the boost in this platform is capable of providing tremendous savings in cost for charging the EV. In this simulation, $46.3 \%$ savings is obtained compared to charging the same amount using a typical charger. Special attention should be given to the Eco mode here, since there is a different comparison in terms of energy value. Here, the value of charged energy using the off-grid system is evaluated at \$3.65. These results are summarized in Table 2 .

Table 2. Modes saving compared to the typical plug and charge strategy.

\begin{tabular}{c|c|c}
\hline \multirow{2}{*}{ Mode } & \multicolumn{2}{|c}{ Saving compared with the typical charger } \\
\cline { 2 - 3 } & On-grid & Off-grid \\
\hline Normal & $30 \%$ & $79.8 \%$ \\
\hline Eco & $58.4 \%$ & $100 \%$ \\
\hline Boost & $0 \%$ & $46.3 \%$ \\
\hline
\end{tabular}

\section{Conclusion}

In this paper, a multi-mode optimization technique is developed to achieve the objective of reducing the cost of the EV battery charging. Several modes were adopted to satisfy customer needs. Normal mode, eco mode, and boost mode are suggested with different features to suit varying customer needs. Simulation results show the effectiveness of this technique, some modes where able to cut the cost by more than 50\%, however, all the savings in the simulation are highly dependent on the initial parameter. Moreover, this technique will put no additional stress on the power system components. On the other hand, it will help to attain a smother load curve by shifting the EV load from on-peak hours to off-peak hours.

\section{References}

[1] Chen Q, Sun F, and Zhu J. Modern Electric Vehicle Technology. Beijing, China: Beijing Institute of Technology Press, 2004.

[2] Yu XE, Xue Y, Sirouspour S, and Emadi A. Microgrid and transportation electrification: A review. 2012 IEEE Transportation Electrification Conference and Expo (ITEC), Dearborn, MI, 2012.

[3] Qin Y, Bei Z, and Mladen K. Optimized Operational Cost Reduction for an EV Charging Station Integrated with Battery Energy Storage and PV generation 2017

[4] Cao Y, Tang S, Li C, Zhang P, Tan Y, Zhang Z, and Li J. An optimized EV charging model considering TOU price and SOC curve. IEEE Transactions on Smart Grid, 2012; 3(1): 388-393.

[5] Etezadi-Amoli M, Choma K, and Stefani J. Rapid-charge electric- vehicle stations. IEEE Trans. Power Del., June 2010; 25(3): 1883-1887.

[6] Jiang $\mathrm{Z}$ and Dougal R. Control strategies for active power sharing in a fuel-cell-powered battery-charging station. IEEE Trans. Ind. Appl., May 2004; 40(3): 917-924.

[7] Zhu X, Chen H, Liu W, and Luo J. Design and exploitation of supervisory control system for commercial electric vehicle charging station based on virtual DPU technology. in Proc. 2010 Power Syst. Technol. (POWERCON) Conf., pp. 1-5.

[8] Kou P, Liang D, Gao L, and Gao F. Stochastic coordination of plug-In electric vehicles and wind turbines in microgrid: A model predictive control approach. IEEE Transactions on Smart Grid, May 2016; 7(3): 1537-1551.

[9] Cao Y, Tang S, Li C, Zhang P, Tan Y, Zhang Z, and Li J. An optimized EV charging model considering TOU price and SOC curve. IEEE Transactions on Smart Grid, 2012; 3(1): 388-393.

[10] ERCOT website www.ercot.org (2018).

[11] California ISO", Caiso.com, 2018. [Online]. Available: http://www.caiso.com. [Accessed: 28- Apr- 2018].

[12] "PVOutput", Pvoutput.org, 2018. [Online]. Available: https://pvoutput.org. [Accessed: 28- Apr- 2018].

[13] "Nissan UK | Electric cars, crossover, 4x4 \& vans", Nissan, 2018. [Online]. Available: https://www.nissan.co.uk. [Accessed:28- Apr- 2018]. 